and the photographer can operate as safely in his car as in his studio. It will also add to the resources of photography, for there are no places on the earth's surface inaccessible to a balloon.

The ascent here described had for its main purpose photography; but it had also some meteorolcgical interest. The ascent began at I.20; and at 3.20, at an altitude of I IOO metres above Meaux, another balloon, which ascended some time after them, was met with. They were-actually in a frequented aëronautical routean aërian river. At Meaux Nadar descended in 1863 ; M. Tissandier himself landed at the same place in 1872 , and sereral other descents were made there. A little farther, at Château-Thierry, on a prolongation of the line from Paris to Meaux, M. Tissandier and M. de Fonvielle made an extraordinary descent in a storm in 1869 , when they were dragged along the ground four kilometres in five minutes. They travelled from Paris to ChâteauThierry, a distance of 80 kilometres as the crow flies, in 35 minutes-the most rapid balloon voyage on record. On the present ascent, at an altitude of 1000 to I 400 metres, an aërial current of considerable speed prevailed ; it was estimated at about 40 kilometres an hour. At 1400 metres a mass of white translucid clouds stretched across the sky and floated in the upper part of the aërial current. Above this, again, the air was calm; small white clouds remained immovable at 2000 metres, and the sun was very hot. After having descended close to the earth above Château-Thierry, it was decided to rise above the clouds amongst which the aëronauts had just been. At 6 o'clock, at a height of 1900 metres, they observed the shadow of the balloon projected on a white ground of clouds; the latter formed a small greyish circle, surrounded by an aureole of the seven colours of the rainbow. When they approached the clouds, it was only the shadow of the car and of the lower part of the balloon of which the projection could be distinguished, and the aureole assumed a larger diameter. This remarkable and beautiful phenomenon resembles that of the spectre of the Brocken. At 6.Io the descent commenced; the balloon crossed the bank of clouds, and the surface of the earth, when it came in sight, looked grey and dull compared with the magnificent regions of the upper atmosphere.

\section{RADIANT LIGHT AND HEAT ${ }^{1}$}

III.

Radiation and Absorption-Terrestrial Applications.

HAVING now established the Theory of Exchanges, let us inquire at greater length into the nature of the radiation from bodies of different kinds. For this purpose we shall adopt the well-known classification into solids, liquids, and gases, and shall select as the type of a solid body (as far as radiation is concerned) a black substance like carbon. We must do this because, in order to obtain the greatest amount of radiation from such a body at a given temperature, it must be of sufficient depth to be practically opaque, or athermanous, for the heat of that temperature, and it must have a non-reflective surface. Now carbon or lamp-black possesses these properties, if not completely, yet to greater perfection than any other substance that we know of; and on this account we shall select it as the type of radiating solid bodies.

Then as regards liquids, we have no doubt an amount of surface-reflexion, which will have the effect of diminishing the radiation, and also of polarising it, to some extent. In this respect a liquid surface may be regarded as equivalent to a polished solid surface, so that liquids and polished solids may be classed together as giving out an amount of heat somewhat lcss than that given out by the typical black surface.

But while there is no marked distinction in radiation I Continued from page 399 . between solids and liquids, if only the depth of substance be sufficiently great, the radiation of gases is essentially different. This difference consists in the fact that while solids and liquids radiate all kinds of heat possible to the temperature, gases radiate only a few. We shall best perceive this distinction if we confine ourselves to rays which affect the eye, and view these by means of the spectroscope.

We have already explained how this instrument draws out a thread of white light into a parti-coloured ribbon, red at the one end and violet at the other. Now if our thread of white light be a thread of platinum, or, better still, of carbon rendered incandescent by means of electricity, we shall no doubt obtain the spectrum above mentioned. But if our source of light be a row of incandescent gaseous particles, we shall obtain something very different. Instead of a long, continuous, variously coloured ribbon, we shall have a few discontinuous threads of light emerging from a dark background, each such thread or image having of course its proper spectral position; that is to say, if the gas gives out a yellow ray, this will appear in the yellow region of the spectrum; if a red ray, in the red region, and so on. Such spectra may either be thrown upon a screen, or viewed through a telescope-sometimes it is possible to throw them upon a screen and render them visible to a large audience, but sometimes this is not possible. In all cases, however, they may be thrown into a telescope and viewed by the individual observer.

We are thus in a position to formulate the distinguishing characteristic between the spectra of solids and liquids, and those of gases, the former giving out a continuous spectrum, consisting of all the rays of light possible to the temperature, while the latter give a discontinuous spectrum, consisting of a few bright lines on a dark background.

We can, in an imperfect manner, assign a reason for this behaviour. In a solid, or even a liquid, the various molecules are near together, so that no individual is free from the trammels of its neighbour in its vibrations. On the other hand, it is not so in a gas, or at least in a gas of which the molecules are very far from one another.

Here onc individual is for the most part of its existence free from the trammcls of its neighbours, and is able to vibrate after its own fashion and in a way to suit itself, just as freely as a bell, or the string of a musical instrument. It thus gives out, as it were, its own peculiar note, or series of notes, these notes being here, however, rays which have a definite place in the spectrum, instead of sounds which have a definite place in the musical scale. But whilst there is a great amount of frcedom amongst the molecules of a gas, we must not carry this conception of things too far, or suppose that in a compound gas at ordinary temperatures we have nothing but a series of perfectly similar molecules practically independent of one another.

The particles or molecules of such a gas are far from being in a state of rest, and we may imagine them to be running about in straight paths, except when they are deflected by dashing against a neighbour, or against the sides of the containing vessel. It will thus be seen that the molecules are not quite free. In fact, a molecule perfectly remote from neighbours, travelling, for instance, in frce space, and remote from the sun, would have no more inducement to vibrate than a bell would have under similar circumstances. It is the collision with its fellows that will generally cause it to vibrate, but it is sufficiently independent to vibrate according to its own laws. Indeed, we are in a position to assert that a great portion of that energy which constitutes ordinary heat in a gas is derived from this motion of the molecules in straight lines, while, again, the radiation of the gas is caused by the vibrations of the molecules after they have been in collision with one another, or with the sides of the containing vessel. 
Now in a compound gas these collisions sometimes cause dissociation of the compound molecule into more elementary constituents, which constituents will probably afterwards combine again, so that we may imagine that in such a gas (see "Heat," by Prof. Tait, page 203) equilibrium is maintained by a constant amount of dissociation, accompanied by an equal amount of recombination. It is thus apparent that we have not here perfect simplicity and uniformity of molecular structure, and without discussing the question whether a simple molecule might or might not be expected to vibrate in only one way, we can readily imagine that the spectrum of such a gas should present us with more than one mode of vibration ; that is to say, more than one spectral line.

Again, circumstances which conduce to proximity of molecules, and to the action of molecules upon each other, tend to bring about a state of things similar to that which we have in liquids and solids; that is to say, they will favour the emission of various kinds of rays, while on the other hand, the characteristics of a gaseous spectrum will be best shown by a perfect gas, that is to say, by a gas which is far removed from any tendency to condensation. A rare gas at a high temperature will possess these properties.

Having now defined the characteristics of the spectra of solids, liquids, and gases, let me say a few words about the methods by which we obtain gaseous particles heated to a high temperature. These are obtained in two ways. First, by means of flames, such as that of a Bunsen's burner, into which the particles are introduced. In such flames we may imagine that we have before us a certain number of the particles of a certain gas all, or nearly all, heated to a temperature somewhat approaching that of the flame. The substance will probably have been introduced into the flame in a different chemical state from that in which it appears in giving out the light; for instance, we may introduce into a spirit-lamp a little chloride of sodium, or into a Bunsen's burner a little bicarbonate of soda. The flame becomes immediately of a yellow nature, giving us the double line $D$, or the yellow line of incandescent sodium vapour, and this affords us evidence that dissociation has taken place. In like manner the red line produced by salts of Lithium, the green line produced by those of Thallium, and so on, are indications that the compound saline molecules have become dissociated in the flame.

The second way of producing gaseous spectra is by an application of electricity, as when a high tension spark is sent through a tube containing a small quantity of a given gas, or a vacuum tube, as this is sometimes called. We have then a momentary flash, consisting of the rays which characterise the spectrum of the gas through which the discharge has passed. It is probable that in this case only a portion of the particles filling the tube have been brought to the high temperature which is denoted by the discharge.

Before proceeding further, it may be well to mention that while from the title of our subject we must necessarily consider the spectrum to some extent, yet this is not to be regarded as a treatise on the spectroscope and its applications, which formed the subject of a previous set of essays in the NATURE Series by Mr. Lockyer. We shall discuss the subject in a somewhat different manner, and also give more especial attention to those branches which had not yet been developed when Mr. Lockyer wrote his work. With these preliminary remarks, we shall divide the subject before us into two sections.

(I) Radiation and its consequences.

(2) Absorption and its consequences.

In the first we shall discuss radiant spectra to a considerable extent, but shall not entirely confine our remarks to these phenomena; while in the second we shall discuss absorption spectra to a considerable extent, but shall not entirely confine ourselves to spectral absorption.
There is likewise another convenient way of dividing our subject, namely, in its application to terrestrial and celestial phenomena.

Combining, therefore, these two principles of sub division, we shall, in the first place, treat of terrestrial applications of the laws of radiation and absorption, and in the next place of their celestial applications; and, finally, we shall discuss the light which both of these branches together appear to throw upon the ultimate constitution of matter.

With regard to our own Earth, it is abundantly evident that the great bulk of the heat which it receives is from the radiation of the sun, while, on the other hand, the great bulk of the heat which it loses is through radiation into space.

There is a sort of balance kept up between the gain on the one hand and the loss on the other, in virtue of which we are placed under conditions in which life is endurable, and for the most part pleasant. The variations in these conditions in temperate latitudes may sometimes cause distress to the weak, but they are not less the source of enjoyment and vigour to the strong ; and, as a matter of fact, the most energetic races of mankind are they which dwell in those favoured regions that are neither too cold nor too hot.

Inasmuch as the regions near the equator are hotter than those near the poles, it follows that there is greater radiation into space from the former of these than from the latter. If, therefore, we could imagine an observer to be placed many thousand miles above the earth, having an eye capable of distinguishing dark rays, and to regard that portion of the earth unilluminated by the sum, his eye would receive more rays from the equatorial than from the polar regions.

On the other hand, the polar regions being manifestly colder than those of the equator, we have convection currents of hot air passing in the upper atmospheric regions from the equator to the poles, and currents of cold air passing in the lower atmospheric regions from the poles to the equator. These latter are known as the Trade Winds, and the former as the Anti-Trades. In like manner we have in all probability currents of hot water passing in the upper oceanic regions from the equator to the poles, and currents of cold water passing in the lower oceanic regions from the poles to the equator. It is not, however, our object to dwell on these phenomena here; suffice it to say, that our well-being depends on the balance between the radiant heat which we receive from the sun and that which we give out into empty space.

The phenomena of dew form an exceedingly good illustration of the laws of radiation. This subject was first investigated by Dr. Wells, an English physician. When the sun has sunk beneath the horizon of any place, bodies of small mass and great radiating power for dark heat, such as the leaves of plants, become quickly cooled by their uncompensated radiation into space. They thus cool the air around them, until this air becomes so cold that it can no longer retain in the viewless state the aqueous vapour which it holds; part of this is consequently deposited in the form of dew, or of hoar-frost, if the temperature be sufficiently low.

The following are the laws which regulate the deposition of dew :-

(I) Dew is most copiously deposited under a clear sky.

(2) And with a calm state of the atmosphere.

(3) It is most copiously deposited on those substances which have a clear view of the sky.

(4) And which are good radiators and of small mass.

(5) And which are placed close to the earth.

The first of these conditions is essential, because the cooling which precedes the deposition of dew is owing to radiation into free space.

If there are clouds, these will radiate back to the body, 
and thus prevent it from cooling fast enough. We see, likewise, the necessity for a calm atmosphere, when we reflect that dew can only be deposited by means of the body cooling the air around it ; now if this air is constantly renewed, it cannot cool this large body of air to any great extent, and hence dew cannot be formed.

It is very manifest why the body must have a clear view of the sky, and why it must be a good radiator in order to promote the deposition of dew. Also why it must not be of a great mass, for, if it were, the heat from the interior might be conducted to the surface, and thus keep up the temperature.

Finally, the substance must be near the earth, for, if not, the cooled air will fall down, giving place to warmer air. The body will thus have a larger mass of air to cool, and it will less easily succeed in bringing this mass below the dew point. I shall return to this subject at a later stage, when the part played by the aqueous vapour of the air is taken into account. Let me here state that there are regions in the earth where dew forms an important factor in agricultural operations.

The artificial warming of our rooms is at present accomplished very much by radiation. An ordinary fire of coal or wood acts by this process. The heated carbonic acid gas which is the product of the combustion is carried up the chimney and out into the air, so that all that remains to heat the room is the light and heat given out by the glowing fire.

It is by no means an economical use of heat, but there are other considerations besides those derived from economy, and an open fire will always be cherished by those nations whose social life is greatly within doors.

The burning of gas in order to obtain illumination has nothing to recommend it. As it is used at present, it gives out a great deal of heat compared to its light, as well as a quantity of carbonic acicl, and other products still more deleterious.

It ought to be replaced by some kind of electric light, such as that proposed by Swan, where a thread of carbon is kept at a high temperature in a glass vacuum by means of an electric current. There the luminous effect is very large in comparison with the heat produced, besides which there is no foul air or other hurtful product.

If we regard radiation as a means of increasing our knowledge, apart altogether from its primary and indispensable action in rendering us acquainted by means of vision with the objects around us, we cannot have a better instance than that which is given us in spectrum analysis. Here, in the first place, a little reflection will convince us that we can gain hardly any knowlege by this means of the nature of a luminous solid or liquid body, for all such bodies at the same temperature will give out all the various rays which are possible to that temperature. There is, therefore, no means afforded us by their spectra of distinguishing one from another, so that spectrum analysis is here impossible.

It is very different, however, when we come to gases which give out spectra consisting of bright lines in a dark background. Here there are various laws which combine not only to make spectrum analysis possible, but to constitute it an extremely delicate instrument of research. In the first place, we have the law that the lines given out by any one clementary vapour are diffcrent in spectral position from those given out by any other. Secondly, as a rule such bright lines remain in their places throughout a great temperature range. Thirdly, an exceedingly small amount of the element in question is generally sufficient to produce the lines.

It is stated that by means of the spectroscope the presence of less than one two-hundred-millionth part $\left(\frac{1}{200,000,000}\right)$ of a grain of sodium may be detected. Indeed, the difficulty is to get rid of the sodium line in an insular climate like ours, surrounded by sea-water which contains chloride of sodium.

There are three chief points for consideration in the study of gaseous spectra:-

(I) The effect produced by increasing the pressure of the gas.

(2) The effect produced by giving the gas a motion to or from the observer.

(3) The effect produced by increasing the temperature of the gas.

The effect produced by increase of pressure consists in a widening of the bright lines. This subject was first studied by Frankland and Lockyer, who found that all lines are not affected by pressure to nearly the same extent. The $\mathrm{F}$ line produced by incandescent hydrogen was found by them to be peculiarly subject to an increase of pressure, widening out in certain cases to a really remarkable extent.

Lockyer, who has since greatly studied this subject, is of opinion that it is not pressure per se that is influential in thickening the lines, but rather the frequency of encounters of precisely similar molecules. An important application of this law of pressure has been made by Lockyer, who has for this purpose used the electric arc, placing the slit of his spectroscope so as to embrace a section of this arc mid-way between its terminals and at right angles to its length. Now in the heart or central axis of this arc the gaseous particles which give out the light may be supposed to be somewhat near together, whereas at the border or circumference they are comparatively far apart. When the spectrum of such a transverse section is taken, this is found to consist of a number of bright lines, some long and some short. The long lines are those which remain visible even when the particles are far apart, while the short lines are those which require a greater nearness of particles to come out, and are therefore confined to the central regions of the arc.

Suppose now that we take the spectrum of such an arc, from terminals composed of absolutely pure iron, and that by this means we obtain a number of long and short lines, characterising the spectrum of this metal in the state of vapour.

Suppose next that we obtain the spectrum of some other metal, such as copper, which is not chemically pure, but which, we suspect, contains a little iron. We shall obtain, of course, the copper lines well defined and intense, plus an indication of the iron lines; but inasmuch as the iron particles are here few and far between, the iron lines which make their appearance will be those which do not require great nearness of particles in order to come out-in other words, they will be the long iron lines, and not the short ones. In searching spectroscopically for an impurity it is thus only necessary to direct our attention to the long lines of the various metals which we suspect to be present. Thus the whole process of comparison is made much simpler, and we are enabled likewise to obtain with comparative ease the true spectra of the various elements.

Let me now say a few words about the effect produced by a motion of the radiating gas to or from the observer. Suppose that a tram car starts from a station every five minutes in a certain direction, and that we are walking briskly towards this station, we shall meet the cars oftener than cvery five minutes. On the other hand, if we are walking briskly from the station, they will overtake us less frequently than every five minutes. Suppose, again, that the whistle of a locomotive engine strikes the air $\mathrm{r}, \mathrm{coO}$ times every second, then if the locomotive be at rest, we know from the theory of sound that the one blow will have advanced about 13 inches before the next is delivered to the air.

If, however, the locomotive engine be itself travelling in this direction, it is evident that the interval between the blows will be less, for the engine may have itself 
advanced one inch during the time that the last blow has advanced 13 inches, and thus the distance between the two blows will be $\mathrm{I} 2$ inches, or one foot. If, therefore, an observer be standing on a railway platform and a railway engine be advancing at full speed whistling as it comes, the interval between the blows will be less than usual, or the note will be shriller than if the engine were at rest. On the other hand, when it has passed the station and is rapidly receding from the observer, the interval will be greater than usual, and the note less shrill.

It is precisely the same with regard to light. If a luminous body emitting rays of definite wave length be moving towards the observer, the wave length will be lessened and the ray pushed forwards to the more refrangible side of the spectrum. If, on the other hand, it be moving from the observer, the wave length will be increased, and the ray pushed backwards to the less refrangible side of the spectrum.

'The only difference between light and sound is that the former moves so fast, that in order to get an appreciable alteration in wave length we must have a luminous body moving from or towards us with velocities much greater than we can produce experimentally, whereas in the case of sound we can make the experiment.

Nevertheless if we go to the surface of the sun, or to the fixed stars, we shall find luminous objects moving from or towards us with velocities sufficiently great to suit our purpose.

Let me now say a few words on the effect produced on some gaseous spectra by increasing the temperature of the gas. It is quite certain that at comparatively low temperatures such spectra are more complicated than they are when the temperature is high. In the former case they frequently present a fluted appearance, while in the latter we have spectra composed of a few bright lines on a dark background.

In some cases an increase of temperature entirely changes the character of the spectrum, so that certain socalled elementary substances may be said to have two or more spectra. In general, however, we have, notwithstanding these remarks, the great feature already mentioned of a persistence of the more permanent spectral lines, more especially in the case of metals, throughout a large temperature range.

By means of spectrum analysis we have discovered the existence of several new elementary metals, all of which are very sparingly distributed.

Bunsen was the first to detect two new elementary metals, coesium and rubidium. Shortly afterwards Crookes discovered thallium, Messrs. Reich and Richter indium, and other elementary metals have since been discovered by the same means.

It is now time that something should be said about the phenomena of absorption. Since gases have small radiating powers, they may naturally be supposed to have small powers of absorption. We know, for instance, how feeble is the absorption of pure air for luminous rays, or even for ordinary heat rays. Tyndall has studied the absorptive power of gases for low temperature heat, and has come to some very interesting conclusions. The following table embodies the results of his experiments:-

Comparative absorption of various gases, each of the pressure of $\mathrm{I}$ inch.

$\begin{array}{lllllllllll}\text { Air } & \ldots & \ldots & \ldots & \ldots & \text { I } & \text { Nitric oxide } & \ldots & \ldots & \text { I590 }\end{array}$

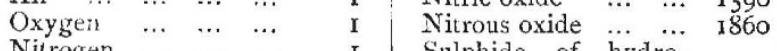
\begin{tabular}{lllll|l} 
Nitrogen & $\ldots$ & $\ldots$ & $\ldots$ & I & Sulphide of hydro-
\end{tabular} \begin{tabular}{ccccc|ccccc} 
Hydrogen & $\ldots$ & $\ldots$ & $\ldots$ & I & gen $\ldots$ & $\ldots$ & $\ldots$ & $\ldots$ & 2 roo \\
Chlorine & $\ldots$ & $\ldots$ & $\ldots$ & 60 & Ammonia & $\ldots$ & $\ldots$ & & 7260
\end{tabular}

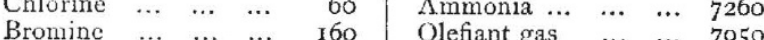
$\begin{array}{lllllll}\text { Hydrobromic acid } & \ldots & 100 & 1005 & \text { Sulphurous acid } & \ldots & 8950 \\ \end{array}$ Carbonic oxide ... $\quad \ldots \quad 75^{\circ}$

By this we learn that the absorptive power of the three permanent simple gases for dark heat is very small, while that of compound gases is very considerable. Tyndall imagines that the molecule of a compound gas may be more inert and less nimble in its vibrations than that of a simple gas. That is to say, the compound molecule will vibrate more slowly than the simple one, and will thus give rise to rays of great wave length; and inasmuch as its absorption and radiation are connected together, it will be peculiarly liable to absorb rays of great wave length.

Its absorption for dark heat may therefore be very great, even although it may appear perfectly transparent for ordinary light rays.

Tyndall has found, as the result of his inquiries, that aqueous vapour absorbs many more dark rays than dry air, and justly concludes that the aqueous vapour present in the atmosphere plays a very important part in terrestrial economy. Being transparent for rays of high temperature it stops but a small proportion of those which come to us from the sun; on the other hand, being comparatively opaque for rays of low temperature, it stops the radiation into space from the surface of the earth. To speak more accurately, it does not absolutely prevent this radiation, but absorbs it and returns as much or nearly as much again. Its action, in fine, is virtually the same as that of a cloud in preventing the refrigeration which accompanies dew. Tyndall remarks that in those regions where the air is very dry the nights are often intolerably cold, owing to this uncompensated radiation into space.

Such regions are those in Central Asia and the great African desert, in the latter of which water can readily be frozen after the sun has sunk. The glass of a greenhouse acts in the same way as the aqueous vapour of the air. It allows the sun's rays freely to penetrate and to heat the air within; but it stops the dark heat of the plants and of the soil from being radiated outwards into free space. Even a loose frame of glass may save the tender blossoms of the peach, and other wall fruit, from being destroyed by nocturnal refrigeration.

\section{BALFOUR STEWART}

\section{(To be continued.)}

\section{NOTES}

ON Monday Prof. Michel Eugene Chevreul entered upon his rooth year. Apart from the fact that among men whose lives have bcen devoted to active scientific research no one has before attained such an age, M. Chevreul stands conspictious for the vast amount of work he has done and for the great practical effect his work has had on the industries of the world. When Dumas in 1852 addressed $M$. Chevreul on the occasion of handing to him the prix of 12,000 francs accorded to him by the Société d'Encouragement pour l'Industrie Nationale, he said :"Le prix consacre l'opinion de l'Europe sur des travaux servent de modèle à tous les chimistes; c'est par centaines des millions qu'il faudrait nombrer les produits qu'on doit à vos découvertes.' More recently, in 1873 , when the award of the Albert medal was made by our Society of Arts, the terms in which the Council expressed the grounds of the award were :- "For his chemical researches, especially in reference to saponification, dyeing, agriculture, and natural history, which for more than half a century have exercised a wide influence on the industrial arts of the world." His scientific work, apart from its commercial outcome, was in this country recognised by the Royal Society as far back as 1826 , when he was clected a foreign associate. In 1857 the Copley medal was awarded to him. Other countrie; have also paid him honour, while the distinctions of his native land have showered upon him. Born in Angers in 1786 (on August 31), where his father was a physician of note, he was but seventeen when he went to Paris to be "manipulateur" in the laboratory of the celebrated Vanquelin. At the age of twenty he published his first chemical paper, and in the next half dozen years he had published more than a score on different subjects. Then began that series of papers (commencing in 1813 ), 